\title{
The stimulatory effects of Stewartia koreana extract on the proliferation and migration of fibroblasts and the wound healing activity of the extract in mice
}

\author{
TAE HOON LEE ${ }^{1 *}$, GUY WILHEM LEE ${ }^{2 *}$, KEUN HYUNG PARK ${ }^{2}$, \\ MOHAMED ANTAR AZIZ MOHAMED ${ }^{2}$, MYUN HO BANG ${ }^{2}$, YUN SOO BAEK ${ }^{2}$,

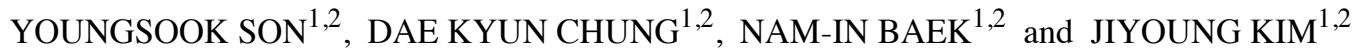 \\ ${ }^{1}$ College of Life Sciences and ${ }^{2}$ Graduate School of Biotechnology, Kyung Hee University, Yongin 446-701, Republic of Korea
}

Received December 9, 2013; Accepted April 17, 2014

DOI: $10.3892 /$ ijmm.2014.1753

\begin{abstract}
Stewartia koreana (S. koreana) has been used in the treatment of inflammatory diseases, such as acute gastroenteritis and aches, in Korean folk medicine and has been reported to have a number of biological activities, such as anti-inflammatory activity and the promotion of angiogenesis. In this study, we aimed to determine the effects of S. koreana extract (SKE) and its components on dermal fibroblast growth and migration, and to investigate the wound healing activity of the extract in mice. In vitro experiments revealed that the numbers of SKE-treated cells increased by approximately 2.5- and 3.7-fold with 50 and $100 \mu \mathrm{g} / \mathrm{ml}$ of SKE, respectively. 5-bromo-2'-deoxy-uridine (BrdU) incorporation was also increased in the SKE-treated cells by 2.3 -fold. SKE promoted the migration of human skin fibroblasts and, among the isolated compounds, hyperin increased the proliferation and migration of the fibroblasts to almost the same degree as SKE. Western blot analysis demonstrated that SKE stimulated the MEK/ERK1/2 and PI3K/Akt signaling pathways. In in vivo experiments, the SKE-treated wound lesions of mice decreased by approximately $7 \%$ in diameter after 2 days of treatment with SKE compared with the wound lesions on the 1st day of the experiment. On the 9th day of treatment, the diameter of the lesions was further reduced by approximately $83 \%$ in the SKE-treated wound areas compared with the wound areas on the 1st day of treatment. Our results demonstrate that methanol extracts of $S$. koreana leaves promote the proliferation and migration of skin fibroblasts and possess effective wound healing activity through the activation of the
\end{abstract}

Correspondence to: Dr Jiyoung Kim, College of Life Sciences and Graduate School of Biotechnology, Kyung Hee University, 1732 Deogyeong-daero, Giheung-gu, Yongin-si, Gyeonggi-do 446-701, Republic of Korea

E-mail: jkim@khu.ac.kr

*Contributed equally

Key words: wound healing, fibroblasts, migration, proliferation, Stewartia koreana
MEK/ERK1/2 and PI3K/Akt signaling pathways. Hyperin was identified as an active compound responsible for the stimulation of fibroblast growth and migration.

\section{Introduction}

Wound healing comprises several phases, such as inflammation, granulation, re-epithelization and tissue remodeling (1). The wound healing processes, include cell migration, simultaneous stimulation of new blood vessel formation, the proliferation of cells, such as keratinocytes and fibroblasts, and the production of basement membrane zones and connective tissues. A number of different cell types are recruited to the wounded skin areas during the healing process (2). After an injury to the skin has occurred, inflammatory cells are attracted to the site of infection to remove the damaged tissues, and then fibroblasts and keratinocytes migrate to the wound lesion and proliferate in order to generate new tissue. Fibroblasts, the most common connective tissue cells, are the predominant cell type in the dermis and play a critical role in wound healing. Fibroblasts produce collagen-based extracellular matrix (ECM) and help reapproximate wound edges through their contractile properties. The remodeling phase, which is a period of consolidation and strengthening, promotes a change in the composition of the ECM of fibers and cells (3).

A variety of herbs and plants have been traditionally used for the treatment of various diseases and wounds in folk medicine in many developing countries (4-6). The choice of herbal products for the treatment of wounds and other ailments depends on the tradition and the plant species grown in different regions of the world. In recent years, many efforts have been made worldwide to discover agents from natural resources that can promote wound healing, reduce the cost of treatment, and save patients from other severe complications (7-9). Stewartia koreana [S. koreana; (Theaceae)], a deciduous tree, is native to Korea, and its tree leaves have been used as a traditional oriental medicine for the prevention and treatment of various diseases. Previous studies have demonstrated that extracts of S. koreana exhibit various biological activities, such as anti-inflammatory activity, the inhibition of osteoclast differentiation and angiogenic activity (10-12). 
In the present study, we analyzed the effects of $S$. koreana extract (SKE) and its isolated compounds on the proliferation and migration of normal human dermal fibroblasts (NHDFs) and evaluated its wound healing activity in mice. We found that SKE stimulated the proliferation and migration of fibroblast cells, and, among the isolated compounds, hyperin was the most effective in stimulating the proliferation and migration of NHDFs. Moreover, we demonstrated that SKE stimulated wound healing in the skin of animals. Our results suggest that SKE is very effective in wound healing, and that hyperin may contribute to the strong wound healing activity of SKE by stimulating the proliferation and migration of normal dermal fibroblasts.

\section{Materials and methods}

Materials and cell culture. Methanol extracts of S. koreana were prepared as previously described (10). Briefly, $S$. koreana leaves were dried and refluxed twice with $80 \%$ methanol. The extract was then filtered and centrifuged at $800 \mathrm{x} \mathrm{g}$ for $10 \mathrm{~min}$. Methanol in the extract was removed under reduced pressure by rotary evaporation and the water residue was removed by lyophilization. The percentage yield was calculated based on the weight of the dried plant material. The dried powder was dissolved in aliquots of DMSO and diluted to the desired concentrations.

NHDFs were cultured in FGM-2 (Clonetics, Lonza, Walkersville, MD, USA) containing $2 \%$ fetal bovine serum (Invitrogen, Carlsbad, CA, USA), 0.1\% recombinant human fibroblast growth factor (rhFGF), $0.1 \%$ insulin (Clonetics), $5 \mathrm{U} / \mathrm{ml}$ of heparin, $100 \mathrm{U} / \mathrm{ml}$ of penicillin and $100 \mu \mathrm{g} / \mathrm{ml}$ of streptomycin (Invitrogen) in a humidified incubator at $37^{\circ} \mathrm{C}$ with $5 \% \mathrm{CO}_{2}$.

Epidermal growth factor (EGF) was obtained from R\&D Systems (Minneapolis, MN, USA) and used as the positive control in each experiment. LY294002 (PI3K inhibitor) and PD98059 (MEK inhibitor) were obtained from R\&D Systems. Other chemicals were obtained commercially from SigmaAldrich (St. Louis, MO, USA).

Isolation and analysis of hyperin in ethyl acetate (EtOAc) extract by high performance liquid chromatography (HPLC). The airdried leaves of $S$. koreana were extracted and fractionated by successive partitioning as previously described (13). Hyperin was obtained from fractions 15 and 16 by further column chromatography of the pooled fraction. For HPLC, the samples were filtered through a $0.45-\mu \mathrm{m}$ microspin PVDF filter. The HPLC equipment used was as follows: an Agilent 1200 series system (Agilent Technology Inc., Santa Clara, CA, USA); G1315 DAD detector, G1329A auto sampler, G1322A degasser, G1311 Quat pump; stationary phase Agilent TC-C18 (5 $\mu \mathrm{m}, 4.6 \times 250 \mathrm{~mm})$; $\lambda=356 \mathrm{~nm}$; binary gradient $\left(\mathrm{A}\right.$, water; $\left.\mathrm{B}, \mathrm{CH}_{3} \mathrm{CN}\right), \mathrm{t}_{0 \text { min }} 50 \%$ $\mathrm{A} \rightarrow$ linear gradient to $15 \% \mathrm{~A}$ within $25 \mathrm{~min} \rightarrow$ linear gradient to $5 \% \mathrm{~A} / 95 \% \mathrm{~B}$ within $14 \mathrm{~min} \rightarrow$ linear gradient to $0 \% \mathrm{~A}$ within $20 \mathrm{~min}$; flow rate $1.0 \mathrm{ml} / \mathrm{min}$; injection volume $1 \mu \mathrm{l}$.

Animal experiments. Animal experiments were approved by the Institutional Review Board (IRB), Kyung Hee University, Seoul, Korea. Healthy BALB/c mice (8 weeks, 25-30 x g) were purchased from Central Laboratory Animal Inc. (Seoul, Korea) and divided randomly into 2 groups (control and SKE-treated group) of 6 mice in each. Each mouse was placed in separate wire-bottom cages in an atmosphere-controlled room $(12 \mathrm{~h}$ light/dark cycle; temperature, $22 \pm 3^{\circ} \mathrm{C}$; and $50 \pm 10 \%$ relative humidity) and maintained on a standard laboratory diet and provided with water.

Wound generation. Mice were anesthetized with $65 \% \mathrm{~N}_{2}$, $30 \% \mathrm{O}_{2}$ and $5 \%$ isoflurane. Anesthesia was maintained with $2 \%$ isoflurane during surgery as previously described (14). Dorsal hair was shaved and wiped with ethanol (70\%) prior to generating the wounds. Two horizontal circular wounds (6 $\mathrm{mm}$ in diameter) were created on the upper back of each mouse using a biopsy punch instrument. Each wound was topically treated either with EGF (R\&D Systems) $200 \mathrm{ng} / \mathrm{mouse}$, PBS or SKE (200 $\mu \mathrm{g} / \mathrm{mouse})$ at the same time each day for 8 days. Images of the wounds were acquired using a digital camera every day immediately before treatment. A small piece of plotting paper $(5 \times 5 \mathrm{~mm})$ was used as a standard for the accurate normalization of the wound sizes recorded in the digital images. Wound contraction was calculated using the percentage of wound size as follows: wound area day $\mathrm{x}$ was divided by wound area day 0 , which was multiplied by 100 (where $\mathrm{x}=1,2,3,,,, \mathrm{n}$, the day after injury).

3-(4,5-Dimethylthiazol-2-yl)-2,5-diphenyltetrazolium bromide (MTT) assay. NHDFs seeded in 96-well plates at a density of $5 \times 10^{3}$ cells/well and allowed to attach for $24 \mathrm{~h}$. After discarding the growth medium, the fibroblasts were treated with SKE or various concentrations of phytochemicals isolated from SKE in serum-free medium for $48 \mathrm{~h}$. EGF (R\&D systems) was used as a positive control. Following incubation, the cells were treated with $100 \mu \mathrm{g} / \mathrm{ml}$ of MTT for $1 \mathrm{~h}$. The formazan precipitate was dissolved in $200 \mu \mathrm{l}$ of DMSO and the absorbance at $560 \mathrm{~nm}$ was determined spectrophotometrically. The analyses were repeated 3 times and the results are expressed as the means of 3 independent experiments.

BrdU incorporation assay. The 5-bromo-2'-deoxy-uridine (BrdU) incorporation assay was performed using BrdU labeling and a detection kit (Roche, Indianapolis, IN, USA) according to the manufacturer's instructions. In brief, the fibroblasts were seeded in 96-well plates for $24 \mathrm{~h}$. After the removal of the medium, the cells were starved for $6 \mathrm{~h}$ and then serum-free medium containing various concentrations of SKE or EGF was added for $24 \mathrm{~h}$. BrdU-integrated DNA was quantified according to the relative luminescence unit (RLU) for each well using a Wallace Victor ${ }^{2} 1420$ multi-label counter (Perkin-Elmer, Monza, Italy). The analyses were repeated 3 times and the results are expressed as the means of 3 independent experiments.

Scratch wound closure assay. The confluent NHDF monolayers in 6-well plates (SPL Inc. Seoul, Korea) were scratched and wounded using a universal sterile $200-\mu 1$ pipette tip and then rinsed with PBS. Each well was treated with various concentrations of medium alone (vehicle), SKE or hyperin for $6 \mathrm{~h}$. EGF was added as a positive control. The cells were stained with Diff-Quick (Baxter Healthcare Corp., McGraw Park, IL, USA) and the width of the wound was measured using an Olympus digital camera (Olympus, Tokyo, Japan). The area of the wound was analyzed using the double-blind direct cell 

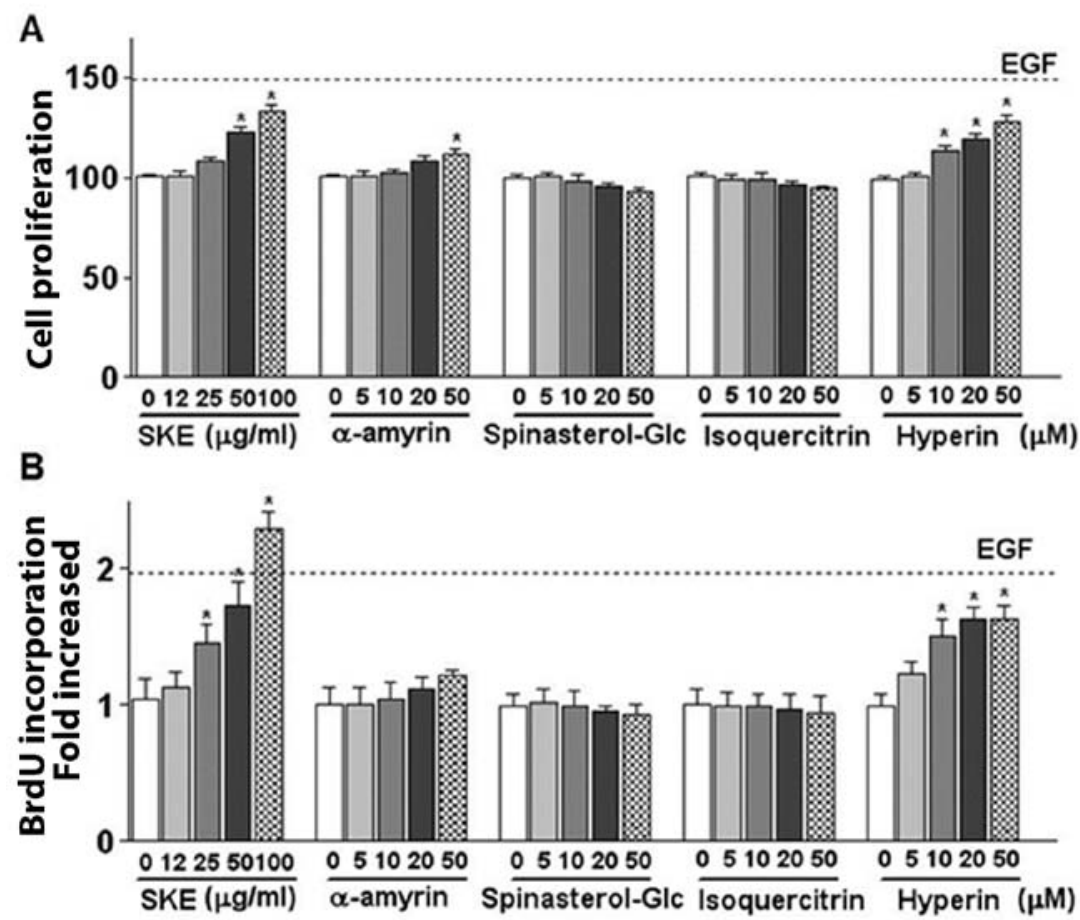

Figure 1. Stewartia koreana (S. koreana) extract (SKE) and its isolated phytochemicals stimulate the proliferation of human dermal fibroblasts. (A) Proliferation of fibroblast cells was measured by MTT assay as described in Materials and methods. Normal human dermal fibroblasts (NHDFs) were plated in 96-well plates in the presence or absence of SKE and isolated phytochemicals, were allowed to attach for $24 \mathrm{~h}$, and were then treated with the indicated concentrations of each sample for $48 \mathrm{~h}$. Cell growth was measured using a spectrophotometer at $560 \mathrm{~nm}$. (B) The rate of relative DNA synthesis of fibroblasts was measured by BrdU incorporation assay. Fibroblasts were plated in 96-well plates in the presence of the indicated concentrations of SKE and isolated phytochemicals. BrdU-integrated DNA was quantified by measuring the relative luminescence unit (RLU). ${ }^{*} p<0.05$ vs. cells in medium alone. Data are shown as the means \pm SD from 3 independent experiments conducted on triplicate samples.

counting method. Each condition was studied in triplicate wells and each experiment was performed 3 times.

Western blot analysis. The cell extracts were prepared from NHDFs treated with various concentrations of SKE or EGF for $15 \mathrm{~min}$. The cells were lysed in RIPA buffer containing proteinase inhibitors (Roche, Indianapolis, IN, USA) and fractionated by electrophoresis on $12 \%$ SDS-PAGE gels and transferred onto a nitrocellulose membrane. Non-specific binding was blocked by soaking the membrane in Tris-buffered saline-Tween-20

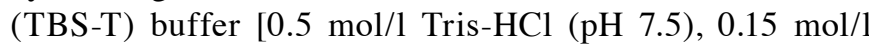
$\mathrm{NaCl}$, and $1 \mathrm{~g} / 1$ Tween-20] containing 3-5\% non-fat dry milk. Phosphorylated (p)-ERK1/2, ERK1/2, p-MEK, MEK, p-PI3K, PI3K, p-Akt and Akt (Cell Signaling, Denvers, MA, USA) were utilized as primary antibodies and peroxidase-conjugated antibody was used as a secondary antibody. The membranes were developed with an enhanced chemiluminescence system from GE Healthcare (Piscataway, NJ, USA) and exposed for $30 \mathrm{sec}$ to X-ray film (Fuji Photo Film Co. Ltd, Tokyo, Japan).

Statistical analysis. The data are presented as the means \pm SD. Statistical comparisons between groups were performed using one-way ANOVA followed by the Student's t-test. A p-value $<0.05$ was considered to be statistically significant..

\section{Results}

Effects of SKE on the proliferation of human dermal fibroblasts. The proliferation of skin fibroblasts is important in tissue repair as fibroblasts are involved in migration, proliferation, contraction and collagen production, leading to the deposition of the ECM and the formation of granulation tissue (1-3). Thus, we investigated the effects of SKE on the proliferation of NHDFs at concentrations up to $100 \mu \mathrm{g} / \mathrm{ml}$ using colorimetric MTT assay. As shown in Fig. 1A, SKE significantly enhanced NHDF growth by approximately $30 \%$ at a concentration of $100 \mu \mathrm{g} / \mathrm{ml}(\mathrm{p}<0.05)$ compared to that of the control untreated cells. In addition, we examined the effects of SKE on DNA synthesis of NHDFs by BrdU incorporation assay. As shown in Fig. 1B, DNA synthesis of NHDFs treated with SKE increased by approximately 2.8-fold compared with the untreated control cells.

Effects of phytochemicals from S. koreana leaves on the proliferation of skin fibroblasts. Previous studies have reported on the isolation of various phytochemicals, such as $\alpha$-amyrin, 3-O- $\beta$-D-glucopyranosyl-spinasterol (spinasterol-Glc), and isoquercitrin from methanol extracts by the successive partitioning of the extracts with EtOAc, n-BuOH (13). Thus, in this study, we examined the effects of isolated compounds, such as spinasterol-Glc, $\alpha$-amyrin and isoquercitrin on the proliferation of skin fibroblasts. Our results revealed that spinasterol-Glc and isoquercitrin did not affect the growth of NHDFs. $\alpha$-amyrin appeared to slightly stimulate the proliferation of NHDFs (Fig. 1A and B), which may not account for the stimulatory effects of SKE. In an attempt to find a compound that stimulates NHDF proliferation, we further fractionated the subfraction originating from fractions 15 and 16 (13), which resulted in the separation of an additional 
A<smiles>O=c1c(O)c(-c2ccc(O)c(O)c2)oc2cc(O)ccc12</smiles>

B

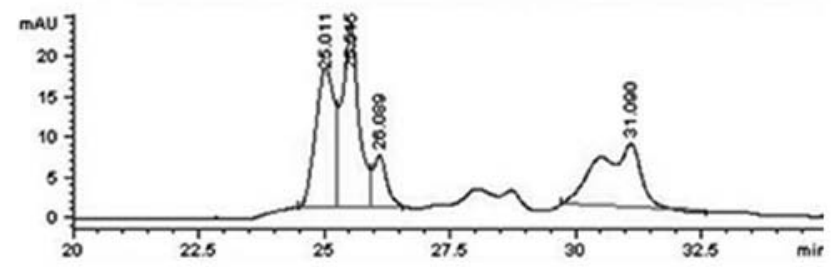

$\mathbf{C}$

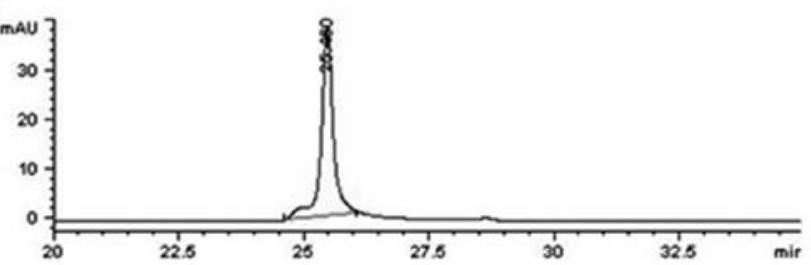

Figure 2. Structure of hyperin and HPLC of EtOAc extract of Stewartia koreana (SKE). (A) Structure of hyperin. In HPLC profiles, the peak appearing at 25.5 min was identified as (B) hyperin in EtOAc SKE, and (C) standard hyperin was detected at $25.5 \mathrm{~min}$. compound. The structure of the compound, which is shown in Fig. 2A, was determined to be hyperin by nuclear magnetic resonance (NMR) spectroscopy (data not shown). HPLC of the EtOAc subfraction revealed that hyperin is a major compound in the fraction (Fig. 2B and C). We evaluated the effects of hyperin on fibroblast proliferation and our results revealed that hyperin strongly stimulated the proliferation of fibroblasts as compared to the control cells (Fig. 1A). Hyperin increased the proliferation of fibroblasts by at least 1.3 -fold and stimulated DNA synthesis by approximately 1.8 -fold at a concentration of $50 \mu \mathrm{M}$ (Fig. 1A and B), which was almost comparable to the effects of SKE.

Effects of SKE and phytochemicals from SKE on the migration of skin fibroblasts. We then investigated the effects of SKE and its phytochemicals on the migration of NHDFs and observed that SKE enhanced the migration of human skin fibroblasts (Fig. 3A and B). When a confluent monolayer of NHDFs was scratched, the migration of cells into the wounded area was significantly increased in the presence of SKE compared to the migration of cells in medium alone $6 \mathrm{~h}$ after wounding. SKE at $100 \mu \mathrm{g} / \mathrm{ml}$ effectively increased the migration of human fibroblasts, which was comparable to the migration induced by EGF. It is therefore plausible that SKE may promote tissue repair not only by increasing proliferation but also enhancing the migration of skin fibroblasts.

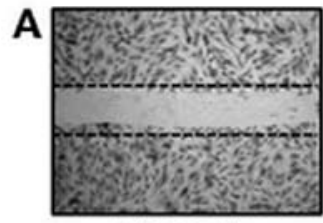

Vehicle

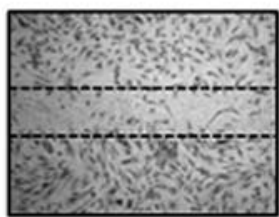

$\alpha$-amyrin

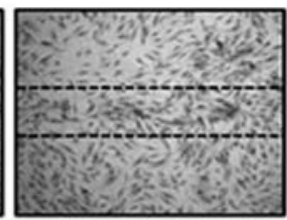

EGF

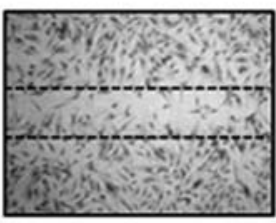

Spinasterol-Glc

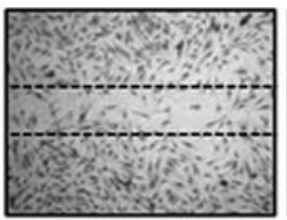

SKE $12.5 \mu \mathrm{g} / \mathrm{ml}$

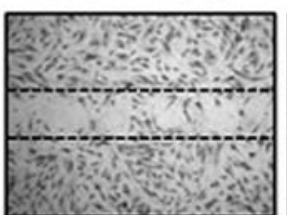

Isoquercitrin

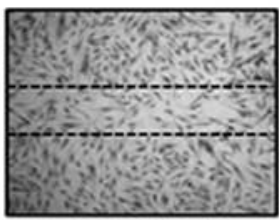

SKE $100 \mu \mathrm{g} / \mathrm{ml}$

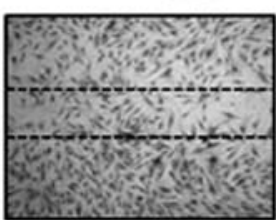

Hyperin

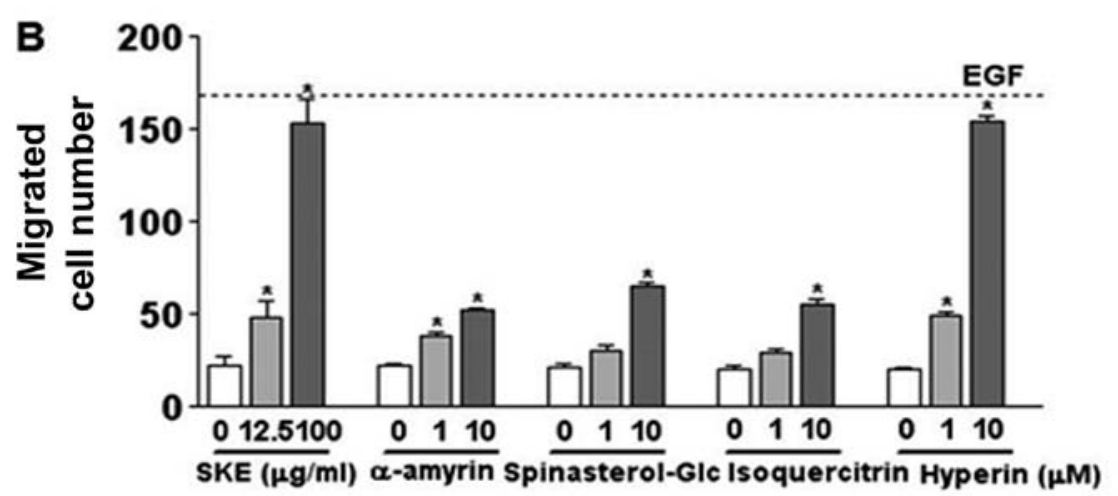

Figure 3. Stewartia koreana (S. koreana) extract (SKE) and its isolated phytochemicals stimulate the migration of human dermal fibroblasts. Human fibroblasts were seeded in 6-well plates. After 24 h, the cells were washed with PBS. (A) Fibroblasts were treated with SKE and isolated phytochemicals at the indicated concentrations for $6 \mathrm{~h}$ and cell migration was detected by wound scratch assays as described in Materials and methods. Representative images of wound scratch assays at the concentration of $12.5,100 \mu \mathrm{g} / \mathrm{ml}$ of SKE and $10 \mu \mathrm{M}$ of each compound, and a positive control (EGF at $10 \mathrm{nM}$ ). (B) The numbers of migrated cells were quantified using double-blind direct cell counting. One representative of 3 experiments is shown. ${ }^{*}<0.05$ compared with cells in medium alone. 
Table I. Effects of the topical application of methanol extract of Stewartia koreana (SKE) on punch wounds on the backs of mice.

\begin{tabular}{lccccr}
\hline & \multicolumn{5}{c}{ Mean lesion diameter $\left(\mathrm{mm}^{2}\right)$} \\
\cline { 2 - 6 } Treatment & Day 0 & Day 2 & Day 4 & Day 6 & Day 8 \\
\hline Vehicle (PBS alone) & $24.77 \pm 2.15$ & $33.15 \pm 3.13$ & $27.18 \pm 3.15$ & $20.71 \pm 2.42$ & $12.11 \pm 1.92$ \\
SKE $(200 \mu \mathrm{g} /$ mice) & $24.86 \pm 2.12$ & $22.53 \pm 3.17$ & $16.25 \pm 2.19$ & $10.02 \pm 1.16$ & $4.34 \pm 0.39$ \\
EGF $(200 \mathrm{ng} /$ mice) & $25.01 \pm 3.21$ & $18.28 \pm 2.76$ & $14.31 \pm 3.01$ & $12.04 \pm 1.72$ & $5.86 \pm 0.64$ \\
\hline
\end{tabular}

SKE $(50 \mu \mathrm{g} / \mathrm{ml})$

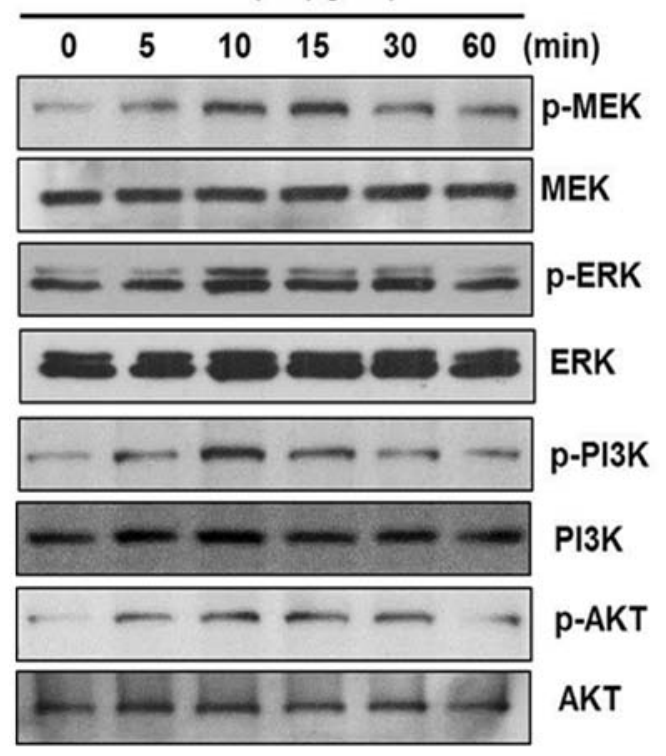

Figure 4. Effects of Stewartia koreana extract (SKE) on the activation of ERK and PI3K signaling pathways. Normal human dermal fibroblasts (NHDFs) were treated with $50 \mu \mathrm{g} / \mathrm{ml} \mathrm{SKE}$ for the indicated periods of time. The phosphorylation state of MEK, ERK1/2, PI3K and Akt was determined with specific antibodies against the phosphorylated form of each protein. The blots were then stripped and re-probed with the antibodies against corresponding MEK, ERK1/2, PI3K and Akt. As the loading control, the level of total protein was measured.

We further examined the effects of its phytochemicals on the migration of fibroblasts by a wound closure assay. Among the compounds tested, hyperin induced the most prominent stimulatory effect on the migration of NHDFs on the wound areas. When a confluent monolayer was scratched, the migration of the cells into the wounded area was markedly increased in the presence of $10 \mu \mathrm{M}$ of hyperin compared to the migration of cells in medium alone $6 \mathrm{~h}$ after wounding. However, $\alpha$-amyrin, spinasterol-Glc and isoquercitrin showed only moderate or very weak activities in promoting the migration of fibroblasts compared to hyperin (Fig. 3A and B). Our results demonstrated that hyperin markedly stimulated fibroblast migration. After $6 \mathrm{~h}$, cell migration was enhanced in response to hyperin $(10 \mu \mathrm{M})$ by 3 - to 5-fold over migration in the presence of medium alone (Fig. 3B). Additionally, the levels of NHDF migration induced by $10 \mu \mathrm{M}$ of hyperin were similar to the those observed for the positive control, EGF $(10 \mathrm{ng} / \mathrm{ml})$. Our results revealed that hyperin stimulated the migration and
DNA synthesis in NHDFs, which promoted wound healing in the animal wound model.

SKE induces the proliferation of fibroblasts through the activation of the mitogen-activated protein kinase (MAPK) and Akt signaling pathways. The activation of MAPK and Akt signaling pathways is known to be involved in the migration and proliferation of skin fibroblasts (15). Thus, we investigated the effects of SKE on the phosphoryration of PI3K/Akt and MAPK/ERK1/2. The serum-deprived NHDFs displayed a barely detectable phosphorylation in the absence of SKE (Fig. 4). However, when SKE was added, ERK1/2, MEK, $\mathrm{PI} 3 \mathrm{~K}$ and Akt were phosphorylated as early as 5 min after the addition of SKE. The phosphorylation reached a maximum level at 10 to $15 \mathrm{~min}$ after exposure, and then decreased. These results suggest that SKE enhances the proliferation and migration of fibroblasts through the activation of the ERK $1 / 2$ and PI3K signaling pathways.

Inhibition of MEK/ERK1/2 and PI3K/Akt decreases SKEinduced proliferation and migration of fibroblasts. To determine the role of the MEK/ERK1/2 and PI3K/Akt signaling cascades in SKE-induced NHDF proliferation and migration, we analyzed the phosphorylation of signaling molecules in the presence of specific signaling inhibitors, such as PI3K inhibitor (LY294002) and MEK inhibitor (PD98059). The SKE-induced ERK activation was inhibited by PD98059, but not by LY294002, indicating that ERK phosphorylation is mediated by SKE-dependent MEK activation. Akt activation was inhibited by LY294002, but not PD98059, suggesting that SKE activates the PI3K/Akt pathway (Fig. 5A). We then examined the effects of these inhibitors on the SKE-induced fibroblast proliferation and migration. The treatment of NHDFs with PD98059 and Ly294002 resulted in a significant decrease in the proliferation and migration of fibroblasts (Fig. 5B and C).

Effects of SKE on wound healing in mice. Wounds on the skin of mice were treated with SKE, EGF or PBS each day, and then wound repair was observed for 8 days. The square measure of each wound was determined on the basis of the pixel number and was then converted to the percentage of wound size. In the SKE-treated mice, wounds healed from a mean area of $24.86 \pm 2.12 \mathrm{~mm}^{2}$ to a mean area of $4.34 \pm 0.39 \mathrm{~mm}^{2}$ (Fig. 6A and Table I). The process of wound healing as a function of time was then depicted as an X-Y plot (Fig. 6B). Eight days after the wound puncture, the wounds were reduced to approximately $83 \%$ of the wound sizes on the first day $(\mathrm{p}<0.05)$ (Fig. 6B). 
A

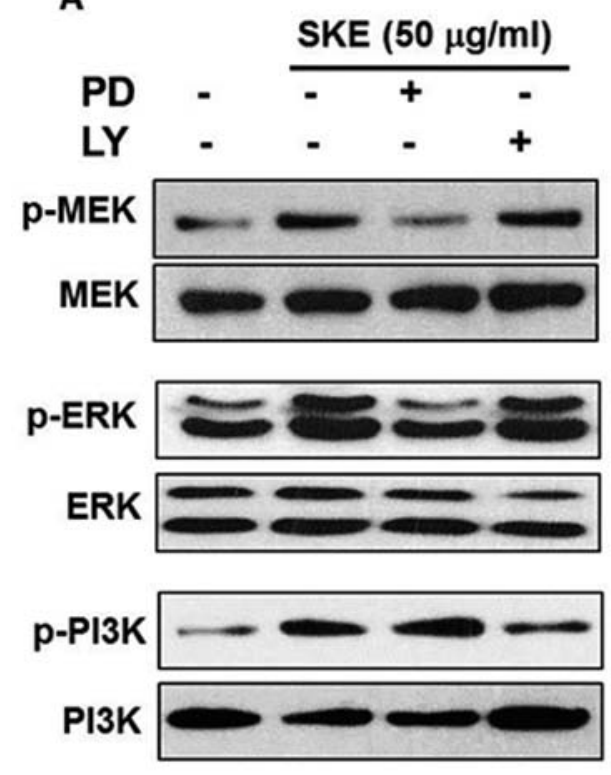

B
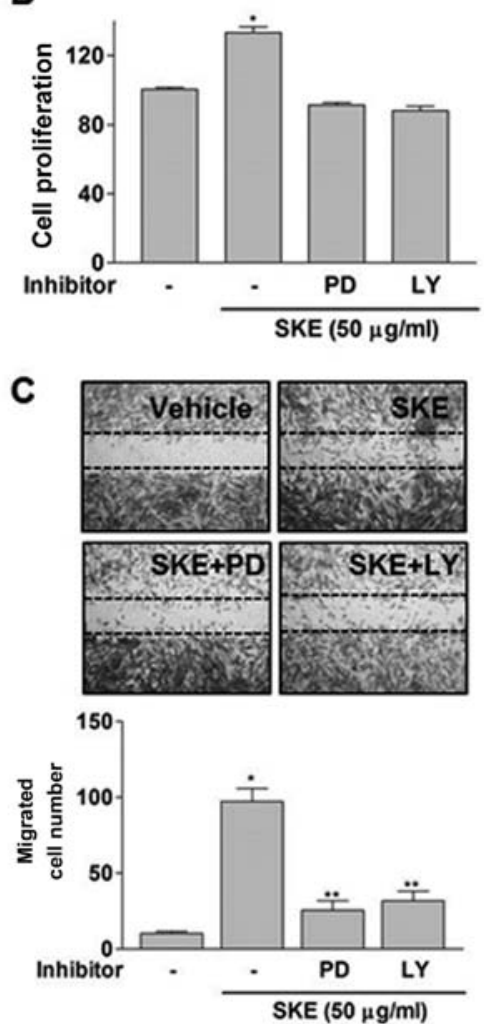

Figure 5. ERK and PI3K signaling pathways are involved in Stewartia koreana extract (SKE)-induced fibroblast proliferation and migration. (A) Normal human dermal fibroblasts (NHDFs) were pre-treated for $1 \mathrm{~h}$ with inhibitors, such as MEK inhibitor (PD98059) and PI3K inhibitor (LY294002) as indicated and then stimulated with SKE $(50 \mu \mathrm{g} / \mathrm{ml})$ for $10 \mathrm{~min}$. Inhibitors were used at the concentration of $10 \mu \mathrm{M}$. (B) NHDFs were pre-treated for $1 \mathrm{~h}$ with specific inhibitors as indicated, and then treated with $50 \mu \mathrm{g} / \mathrm{ml} \mathrm{SKE}$ for $48 \mathrm{~h}$. Cell growth was measured by MTT assay using a spectrophotometer at $560 \mathrm{~nm}$. " p $<0.05$ compared with negative control values (untreated cells). (C) NHDFs were pre-treated for $1 \mathrm{~h}$ with specific inhibitors as indicated. The results of scratch wound assays were photographed (upper panel). The migrated cell numbers were determined by direct cell counting by double blind test (lower panel). Data shown are the means $\pm \mathrm{SD}(\mathrm{n}=3)$. ${ }^{*} \mathrm{p}<0.05$ compared with negative control values (medium alone, vehicle). * ${ }^{* *}<0.05$ compared with only SKE-treated control values. PD, PD98059; LY, LY294002.
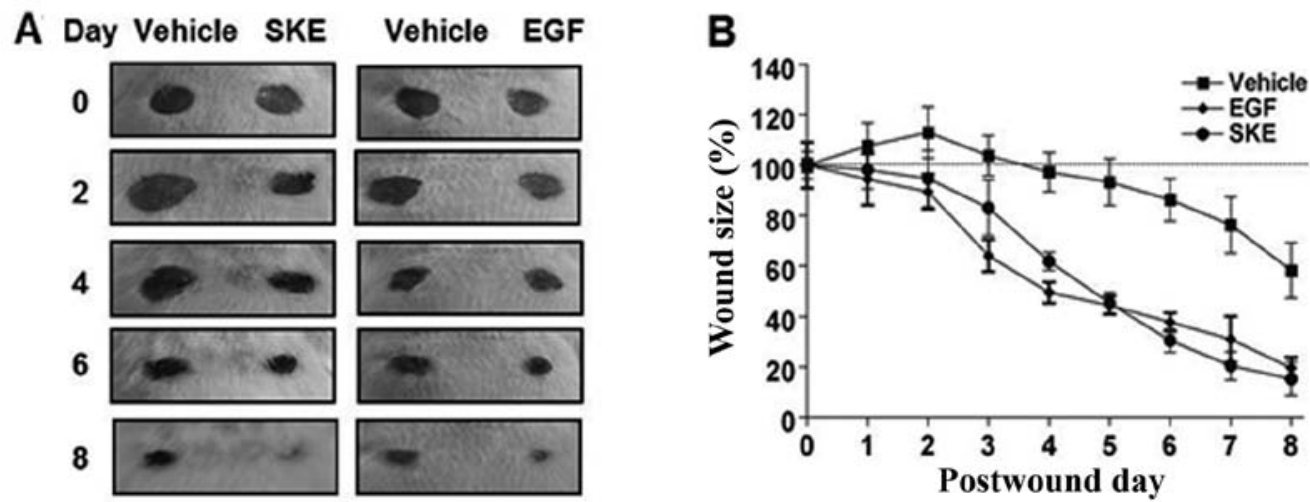

Figure 6. Topical application of Stewartia koreana extract (SKE) accelerates wound healing in a punch wound model on the backs of mice. (A) Representative images of mice with baseline wounds. Digital images obtained during the 8 days of the healing process are shown. SKE (200 $\mu \mathrm{g} / \mathrm{ml}) \mathrm{and} \mathrm{EGF} \mathrm{(200} \mathrm{ng/ml)} \mathrm{were}$ applied every $24 \mathrm{~h}$. (B) The square measures of all 12 mice of both the PBS (vehicle)- and SKE-treated groups were precisely measured by pixel and are represented as the percentage of wound size over time. All data are presented as the means $\pm \mathrm{SE}$.

The wound healing activity of SKE was comparable to that of EGF at the concentrations indicated. The wound sizes of SKE-treated skin markedly decreased compared to the wound sizes of PBS-treated skin, indicating that SKE accelerates wound healing in vivo.
Our data demonstrated that the healing process of the SKE-treated wounds was accelerated after 3 days, when epithelization and provisional matrix formation begins $(1,3)$. The topical application of SKE enhanced cutaneous wound healing, which appeared to be completed in 8 days. Our results showed 
that the original tissue regeneration was much greater in skin wounds treated with SKE than in the control wounds. Moreover, the healing activity of SKE was almost comparable to that of $\mathrm{EGF}$, which is known to accelerate the wound healing process.

\section{Discussion}

A number of studies have reported that various plant extracts contain wound healing activities $(7-9,16,17)$. Skin cell behaviors, such as the spreading and migration of fibroblasts, are major determinants of the wound closure rate. In the present study, we demonstrate that SKE stimulates the growth and migration of NHDFs. We also demonstrate that, among the SKE isolated compounds from the methanol extract of SKE, hyperin was the one that most strongly stimulated the growth and migration of fibroblastss. Our results demonstrated that the SKE-induced fibroblast proliferation and migration were mediated by the ERK and PI3K signaling pathways. Furthermore, we showed that the topical application of SKE enhanced the cutaneous wound healing, which appeared to be completed in 8 days. The healing process of the SKE-treated wounds was accelerated after 3 days, when epithelization, angiogenesis and provisional matrix formation began (1). Our results revealed that the original tissue regeneration was much greater in the skin wounds treated with SKE than in the control wounds, and that the healing activity of SKE was comparable to that of EGF.

The stimulatory effects of SKE on fibroblast proliferation and migration seem to be mediated by MEK/ERK, as well as the PI3K/Akt signaling pathways. The phosphorylation of MEK/ERK and PI3K/Akt were barely detectable under the serum-free conditions and the phosphorylation of these molecules was increased after the treatment of fibroblasts with SKE. The inhibition of the ERK pathway by PD98059 and of the PI3K pathway by LY294002 markedly reduced fibroblast proliferation and migration, indicating that the ERK and PI3K pathways are involved in the SKE-induced cell growth and migration. Our results are consistent with those of previous studies, suggesting that the phosphorylation of Akt and ERK1/2 signaling pathways plays an important role in the promotion of proliferation and migration of fibroblasts $(15,18,19)$. The major function of the ERK and PI3K signaling pathways is to stimulate gene transcription involved in proliferation and migration of cells, including fibroblasts $(18,19)$.

We previously isolated a number of compounds from SKE, including $\alpha$-amyrin, isoquercitrin and spinasterol-Glc. $\alpha$-amyrin belongs to the triterpenoid family (13), which is known to promote ECM accumulation and have an anti-inflammatory activity (20-22). $\alpha$-amyrin is one of the most abundant substances in SKE and is presumed to contribute to the wound healing activity of SKE by suppressing inflammation in the wounded areas and enhancing the production of ECM; however, in this study, it did not promote the proliferation and migration of fibroblasts. Spinasterol-Glc has been shown to strongly inhibit the production of nitric oxide (NO) and proinflammatory cytokines in lipopolysaccharide (LPS)stimulated macrophages (23). Furthermore, spinasterol-Glc promotes the production of procollagen in UVB-irradiated human dermal fibroblast cells (13), and suppresses the production of thymuns- and activation-regulated chemokine (TARC/
CCL17) in human keratinocytes (24). Previous studies have reported that isoquercitrin exhibits anti-inflammatory and antioxidant activities (25).

In the present study, we isolated hyperin (also known as hyperoside or quercetin 3-O- $\beta$-D-galactoside) from SKE and showed that it stimulated the proliferation and migration of dermal fibroblasts in a dose-dependent manner. Hyperin belongs to the flavonoid family, which is mainly found in Hypericum perforatum L. (26). It has been previously isolated from various plants and demonstrated to exert multiple bioactivites, including protection from oxidative damage (27), protective effects against neurotoxicity induced by amyloid $\beta$-protein (28), and both anti-inflammatory and anti-fungal activities (29-31). The wound healing activities of the Hypericum perforatum L. extract have been demonstrated in cultured NIH3T3 fibroblasts and in in vivo animal models $(32,33)$. However, to the best of our knowledge, the biological activity of hyperin in stimulating dermal fibroblasts has not been demonstrated to date. Our study demonstrated that hyperin promoted the proliferation and migration of fibroblast, indicating that it can contribute to the wound healing acitivites of plant extracts, such as S. koreana. Taken together, our data demonstrate that $S$. koreana leaves contain various phytochemicals, including hyperin, spinasterol-Glc, $\alpha$-amyrin and isoquercitrin, which are beneficial for skin wound healing.

In conclusion, this study demonstrates that SKE promotes the proliferation and migration of human fibroblasts in vitro, which may contribute to the enhancement of the wound healing process. Consequently, we identified hyperin as an active constituent and a major promoter of fibroblast proliferation and migration. The methanol extracts of leaves from S. koreana enhanced skin wound healing in an animal model. Thus, $S$. koreana leaves and their isolated compound, hyperin, may be potentially useful for the treatment of wounds and for the development of agents contributing to the topical treatment of wounds.

\section{Acknowledgements}

This study was supported by the Kyung Hee University (S\&P project: 20120726 and sabbatical year project: 20101559), Republic of Korea.

\section{References}

1. Baum CL and Arpey CJ: Normal cutaneous wound healing: clinical correlation with cellular and molecular events. Dermatol Surg 31: 674-686, 2005

2. Werner S, Krieg T and Smola H: Keratinocyte-fibroblast interactions in wound healing. J Invest Dermatol 127: 998-1008, 2007.

3. Brainman-Wiksman LB, Solomonik I, Spira R and Tennenbaum T: Novel insights into wound healing sequence of events. Toxicol Pathol 35: 767-779, 2007.

4. Grabley $\mathrm{S}$ and Thiericke R: Bioactive agents from natural sources: trends in discovery and application. Adv Biochem Eng Biotechnol 64: 101-154, 1999.

5. Biswas TK and Mukherjee B: Plant medicines of Indian origin for wound healing activity: a review. Int J Low Extrem Wounds 2: 25-39, 2003.

6. Davis SC and Perez R: Cosmeceuticals and natural products: wound healing. Clin Dermatol 27: 502-506, 2009.

7. Adetutu A, Morgan WA and Corcoran O: Ethnopharmacological survey and in vitro evaluation of wound-healing plants used in South-western Nigeria. J Ethnopharmacol 137: 50-56, 2011. 
8. Agyare C, Lechtenberg M, Deters A, Petereit F and Hensel A: Ellagitannins from Phyllanthus muellerianus (Kuntze) Exell: Geraniin and furosin stimulate cellular activity, differentiation and collagen synthesis of human skin keratinocytes and dermal fibroblasts. Phytomedicine 18: 617-624, 2011.

9. Hayouni EA, Miled K, Boubaker S, et al: Hydroalcholic extract based-ointment from Punica granatum L. peels with enhanced in vivo healing potential on dermal wounds. Phytomedicine 18: 976-984, 2011

10. Lee TH, Kwak HB, Kim HH, et al: Methanol extracts of Stewartia koreana inhibit cyclooxygenase-2 (COX-2) and inducible nitric oxide synthase (iNOS) gene expression by blocking NF-kappaB transactivation in LPS-activated RAW 264.7 cells. Mol Cells 23: 398-404, 2007.

11. Park CK, Kim HJ, Kwak HB, et al: Inhibitory effects of Stewartia koreana on osteoclast differentiation and bone resorption. Int Immunopharmacol 7: 1507-1516, 2007.

12. Lee TH, Lee GW, Kim CW, et al: Stewartia koreana extract stimulates proliferation and migration of human endothelial cells and induces neovasculization in vivo. Phytother Res 24: 20-25, 2010.

13. Lee TH, Lee SM, Lee DY, et al: A glycosidic spinasterol from Koreana Stewartia promotes procollagen production and inhibits matrix metalloproteinase-1 expression in UVB-irradiated human dermal fibroblasts. Biol Pharm Bull 34: 768-773, 2011.

14. Safer JD, Crawford TM and Holick MF: Topical thyroid hormone accelerates wound healing in mice. Endocrinology 146 4425-4430, 2005.

15. Etscheid $\mathrm{M}$, Beer $\mathrm{N}$ and Dodt $\mathrm{J}$ : The hyaluronan-binding protease upregulates ERK1/2 and PI3K/Akt signalling pathways in fibroblasts and stimulates cell proliferation and migration. Cell Signal 17: 1486-1494, 2005.

16. Balekar N, Nakpheng T, Katkam NG and Srichana T: Wound healing activity of ent-kaura-9(11),16-dien-19-oic acid isolated from Wedelia trilobata (L.) leaves. Phytomedicine 19: 1178-1184, 2012.

17. Zhang Q, Fong CC, Yu WK, et al: Herbal formula Astragali Radix and Rehmanniae Radix exerted wound healing effect on human skin fibroblast cell line Hs27 via the activation of transformation growth factor (TGF- $\beta$ ) pathway and promoting extracellular matrix (ECM) deposition. Phytomedicine 20: 9-16, 2012.

18. Pericacho M, Velasco S, Prieto M, et al: Endoglin haploinsufficiency promotes fibroblast accumulation during wound healing through Akt activation. PLoS One 8: e54687, 2013.

19. Sepe L, Ferrari MC, Cantarella C, Fioretti F and Paolella G: Ras activated ERK and PI3K pathways differentially affect directional movement of cultured fibroblasts. Cell Physiol Biochem 31: 123-142, 2013

20. Maquart FX, Chastang F, Simeon A, et al: Triterpenes from Centella asiatica stimulate extracellular matrix accumulation in rat experimental wounds. Eur J Dermatol 9: 289-296, 1999.
21. Biskup E, Gołebiowski M, Gniadecki R, Stepnowski P and Łojkowska E: Triterpenoid $\alpha$-amyrin stimulates proliferation of human keratinocytes but does not protect them against UVB damage. Acta Biochim Pol 59: 255-260, 2012.

22. Matos I, Bento AF, Marcon R, Claudino RF and Calixto JB: Preventive and therapeutic oral administration of the pentacyclic triterpene $\alpha, \beta$-amyrin ameliorates dextran sulfate sodium-induced colitis in mice: the relevance of cannabinoid system. Mol Immunol 54: 482-492, 2013.

23. Lee TH, Jung M, Bang MH, Chung DK and Kim J: Inhibitory effects of a spinasterol glycoside on lipopolysaccharide-induced production of nitric oxide and proinflammatory cytokines via down-regulating MAP kinase pathways and NF- $\kappa \mathrm{B}$ activation in RAW264.7 macrophage cells. Int Immunopharmacol 13: 264-270, 2012

24. Jung M, Lee TH, Bang MH, et al: Suppression of thymus- and activation-regulated chemokine (TARC/CCL17) production by $3-\mathrm{O}-\beta-\mathrm{D}$-glucopyanosylspinasterol via blocking NF- $\kappa \mathrm{B}$ and STAT1 signaling pathways in TNF- $\alpha$ and IFN- $\gamma$-induced HaCaT keratinocytes. Biochem Biophys Res Commun 427: 236-241, 2012.

25. Rogerio AP, Dora CL, Andrade EL, et al: Anti-inflammatory effect of quercetin-loaded microemulsion in the airways allergic inflammatory model in mice. Pharmacol Res 61: 288-297, 2009.

26. Zou Y, Lu Y and Wei D: Antioxidant activity of a flavonoid-rich extract of Hypericum perforatum L. in vitro. J Agric Food Chem 52: 5032-5039, 2004.

27. Piao MJ, Kang KA, Zhang R, et al: Hyperoside prevents oxidative damage induced by hydrogen peroxide in lung fibroblast cells via an antioxidant effect. Biochim Biophys Acta 1780: 1448-1457, 2008.

28. Zeng KW, Wang XM, Ko H, et al: Hyperoside protects primary rat cortical neurons from neurotoxicity induced by amyloid $\beta$-protein via the $\mathrm{PI} 3 \mathrm{~K} / \mathrm{Akt} / \mathrm{Bad} / \mathrm{Bcl}(\mathrm{XL})$-regulated mitochondrial apoptotic pathway. Eur J Pharmacol 67: 45-55, 2011.

29. Lee S, Jung SH, Lee YS, et al: Antiinflammatory activity of hyperin from Acanthopanax chiisanensis roots. Arch Pharm Res 27: 628-632, 2004

30. Li S, Zhang Z, Cain A, et al: Antifungal activity of camptothecin, trifolin, and hyperoside isolated from Camptotheca acuminata. J Agric Food Chem 53: 32-37, 2005

31. Lee S, Park HS, Notsu Y, et al: Effects of hyperin, isoquercitrin and quercetin on lipopolysaccharide-induced nitrite production in rat peritoneal macrophages. Phytother Res 22: 1552-1556, 2008.

32. Süntar IP, Akkol EK, Yilmazer D, et al: Investigations on the in vivo wound healing potential of Hypericum perforatum $\mathrm{L}$. J Ethnopharmacol 127: 468-477, 2010.

33. Dikmen M, Oztürk Y, Sagratini G, et al: Evaluation of the wound healing potentials of two subspecies of Hypericum perforatum on cultured NIH3T3 fibroblasts. Phytother Res 25: 208-214, 2011. 